\title{
GENETICS, PHYSIOLOGY AND CYTOLOGY OF YEAST-MYCELIAL DIMORPHISM IN FISSION YEASTS
}

\author{
M. SiPICZKI, ÁGNES GRALlERT, IDA MiKLÓS, ERIKA ZILAHI, ANIKÓ BOZSIK \\ and Z. SZILÁGYI
}

Department of Genetics and Institute of Biology, University of Debrecen, Hungary

The order Schizosaccharomycetales contains a dimorphic and two yeast species. Sch. japonicus can form both yeast cells and mycelium, depending on the substrate and the culturing conditions. Sch. pombe is a strictly unicellular organism, but it can be forced to form mycelial cell chains by inactivating members of the sep gene family. The mutations in most of the sep genes confer pleitropic phenotypes indicating functional involvement in MAP-kinasemediated signalling pathways. Two of them were found to encode transcription factor homologues of other eukaryotes.

The fission yeasts are unicellular ascomycetes that are becoming increasingly popular as model organisms for molecular genetical and cytological studies. They comprise an order (Schizosaccharomycetales) with a single family (Schizosaccharomycetaceae) which contains three species: the four-spored Schizosaccharomyces pombe and the eight-spored Sch. octosporus and Sch. japonicus. The four-spored species has two varieties such as Sch. pombe var. pombe and Sch. pombe var. malidevorans. Sch. japonicus is also subdivided: Sch. japonicus var. japonicus, Sch. japonicus var. versatilis and Sch. japonicus var. longobardus (for a review see [1]). Sequence comparison of rRNAs, tRNAs and phylogenetically conserved proteins revealed that the lineage leading to the present-day fission yeasts must have separated from the lineage of the Saccharomyces-type budding yeasts some 1000 million years ago, soon after the Ascomycota separated from the lineage leading to the Metazoa [1]. The phylogenetic gap that separates the two groups of yeasts now is almost as wide as the gaps that separate them from the metazoans. The common ancestor of the fission yeasts is supposed to have been a filamentous fungus that colonized solid substrates but could also fragment into arthroconidia. During a later adaptation to fluid environment, the fragmented (single-cell) form has gradually become the dominating growth morphology

MÁTyÁs SiPICZKi, ÁGNes GRALLERT, IDA MiKLÓs

Department of Genetics, Faculty of Science, University of Debrecen

P.O. Box 56, H-4010 Debrecen, Hungary

ERIKA ZILAHI, ANIKÓ BOZSIK, ZsOLT SZILÁGYI

Institute of Biology, Medical School, University of Debrecen

P.O. Box 1, H-4012 Debrecen, Hungary 
[2]. Sch. japonicus is still dimorphic, because it has retained the ability to form invasive true mycelia on solid substrates [3]. The other two species propagate as yeasts both in liquid and on solid substrates, although $S c h$. pombe can be forced to form mycelium by mutations in certain genes [4-6]. The yeast cells of Sch. pombe and Sch. japonicus have elongated, cylindrical form and grow by polar extension (for a review see [7]). Cell division begins with the formation of a trilaminar septum halfway between the poles (septation) and concludes with the degradation of the central layer of the mature septum (cell separation or cytokinesis) [8]. The polar growth and the dimorphic transitions make the fission yeasts an excellent model for the investigation of cell polarity, one of the basic phenomenon of all living organisms.

\section{The environ mentally controlled dimorphic cycle of $S$ ch. japonicus}

Both varieties of Sch. japonicus can alternate between yeast and mycelial morphology. On solid media, the yeast phase is unstable and can switch to mycelial growth [3]. The transition is induced by starvation of nitrogen, and the growth of the hyphae is directed by the gradients of the nitrogen-containing nutrients in the substrate. The mycelial phase is not stable either; the mycelium can fragment into arthroconidia or return to the yeast form of growth in response to environmental changes [3]. The yeast-tomycelium transition involves a changeover to unipolar growth associated with asymmetric division, the development of large polarly located vacuoles, the reorganisation of the actin and microtubular cytoskeleton and the repression of cell separation after septation [9]. In the yeast phase, the cells usually grow at both poles, whereas the mycelial cells extend only unipolarly, either at the old end (the end that has already grown as the end of the mother cell) or at the new end (the end formed from the septum at the division of the mother cell) [9]. Several lines of evidence suggest that the morphological transitions are cAMP-regulated. For example, the supplementation of the growth medium with caffeine or with cAMP inhibits the yeast-to-mycelium transition and promotes the mycelium-toyeast conversion [9].

\section{Polar growth of $S c h$. pombe cells}

No physiological conditions are known that could be induce mycelial growth in Sch. pombe. Its polarly extending cylindrical yeast cells divide by forming medial septa and then separate to form two physically independent yeast cells (for a review see [7]). In exponentially growing cultures, cell extension is usually initiated from the old end. The new end starts growing much later, at the point in G2 called NETO (new end take off) [10]. However, growth can also start from the new end or from both ends simultaneously as shown by time-lapse photomicrography $[11,12]$. Interestingly, caffeine, that elevates the intracellular cAMP level by inhibiting the degradation of cAMP, makes cell growth more unipolar because it extends the pre-NETO part of cell extension [13]. However, it cannot cause a complete transition to unipolar growth and cannot block cell separation 
either. Thus, the caffeine-treatment drastically changes the growth pattern but cannot change the yeast morphology to mycelial.

\section{Mycelial mutants in Sch. pombe}

Mycelial morphology can be provoked in Sch. pombe by mutations in genes which directly or indirectly participate in cell separation (cytokinesis). The first mycelial mutant identified defined a novel gene, $\operatorname{sep}^{+}$[4]. The $\operatorname{sep}^{-}$cells formed septa (could divide) but did not undergo cell separation (could not cleave the septum) and thus developed cell chains morphologically similar to mycelium. Unlike the transient mycelial phase of Sch. japonicus, the sep $1^{-}$mycelium was stable and could not revert to yeast morphology. The description of sep $1^{+}$was followed by the identification of eleven more sep genes $[5,6]$. In spite of the mycelial growth, the mutant cells retained the bipolar extension pattern, which is also an important difference from the Sch. japonicus mycelium. Since the cell poles were usually covered by unsplit septal material, cell extension could take place only from subapical positions (at both cell ends) and in lateral directions. Thus, the mycelium formed was usually highly branched. This bipolar branching pattern and the lack of vacuolation in the hyphal cells indicate that the $S c h$. pombe mycelium is not an equivalent of the mycelial phase of Sch. japonicus.

\section{sep $1^{+}$encodes a homologue of HNF3 transcription regulator and interacts with M-phase genes}

sep $1^{+}$has been cloned and found to encode a homologue of the HNF-3/forkhead family of tissue-specific and developmental gene regulators common in higher eukaryotes [14]. The aminoacid sequence of the conserved DNA-binding region of the Sep1 protein showed $56 \%$ homology with the corresponding region of the human HNF-3 protein.

sep $1^{-}$synthetically interacts with early mitotic and cell division mutations, such as wee $1^{-}, c d c 2-1 w, c d c 25-22$ and $c d c 4-8[4,15,16]$, which suggests that the Sep1 protein is not involved in cell separation per se, but in some early event(s) of division initiation and therefore its inactivation causes a delay of the whole process of division and shifts its last event, the separation of daughter cells, out of the cell cycle. The inactivation of wee $1^{+}$in sep $1^{-}$background had a peculiar effect: the cells frequently skipped cell division and entered a new cell cycle without septation. The wee $1^{-}$sep $1^{-}$double mutants formed dikaryotic cells at high frequency [16] Thus, sep $1^{+}$seems to be a positive, but indirect regulator of cell separation. 


\section{Contribution of the analysis of cytoskeleton in mycelial mutants to the better understanding of the determination of cell polarity}

The altered cell morphology of the intrafilamental sep- cells provided a possibility to address the role of the microtubular cytoskeleton in the determination of cell polarity. Based on the correlation between the defects of the cytoplasmic microtubules and the deformations of the cell shape, it has been hypothesized that the interphase microtubules play a central role in the determination of the growing poles [17]. The analysis of the reorganisation of the microtubules after cell division in the branched sep $^{-}$cells, however, revealed that they are directed by the cell shape "inherited" from the mother cell. This dependence on cell shape was particularly obvious in the multipolarly growing syncytial cells of the $\operatorname{sep}^{-} \mathrm{spl1}^{-} \mathrm{cdc4-8}$ triple mutant [15]. Furthermore, cell extension cannot be initiated at regions where the cell wall is covered by septal material [16], suggesting that there must exist some communication between the cytoskeleton and the cell wall before establishing a growing site. Thus, the microtubular array is only one member in a multicomponent mechanism responsible for the positioning of the growing cell poles $[15,16]$.

\section{$\operatorname{sep} 2^{+}$is involved in cell separation and division site positioning}

The rest of the sep gene family also seems to have complex functions. The sep2$S A 2$ mutant cells frequently form twin septa separated by anucleate minicells [5]. The longer the cells are, the more minicells can be formed. Presumably $\operatorname{sep} 2^{+}$is required not only for cell separation but also for the correct placement of the division plane. The dependence of the number of septa (division planes) on the lengths of the cell further suggests that there must be a polarly generated intracellular signal which forms a gradient decreasing towards the cell middle. Where it falls below a critical value, a septum is formed. sep2-SA2 might be impaired in the production of this signal [5]. The supposition of the existence of such a polar signal is in conflict with the model proposed by Chang and Nurse [17]. They suggest that the division plane is positioned only by the position of the premitotic nucleus. The polar-oriented signalling mechanism which we suppose to exist in Sch. pombe might be the equivalent of that believed to determine the position of the cleavage furrow in mammalian cells [18]

\section{Mutations in sep6 to sep16 confer complex phenotypes reminiscent of defects in signal transduction pathways}

The mutants sep6 to sep 16 showed not only aberrant cell separation but also impaired sexual activity [6]. None of the mutants could conjugate normally and three of them were also defective in meiosis-sporulation. The examination of the production of sexual pheromones by halo-test revealed that all of them could produce M-factor, but $\operatorname{sep} 8^{-}, \operatorname{sep} 11^{-}$and $\operatorname{sep} 15^{-}$were defective in P-factor production. The mutations in $\operatorname{sep} 8^{+}$, 
sep $11^{+}$and sep $16^{+}$suppressed the pat1-114-driven haploid meiosis, the forced entry into meiosis provoked by the inactivation of Pat1, a negative regulator of meiosis initiation [19].

In addition to the defects in cell separation and sexual differentiation, the sep $6^{-}$to sep $16^{-}$mutants are all super-sensitive to the presence of higher concentrations of chloride in the medium and to short heat shocks [6]. These pleiotropic effects suggest that this group of sep genes might act in or interact with a multiple overlapping network of regulatory modules. Mutations in genes of MAPK-mediated signalling pathways frequently show similarly complex phenotypes. For example, in the budding yeast Saccharomyces cerevisiae signalling pathways have been described which participate in the regulation of pseudohyphal growth, cytokinesis, hyperosmotic stress response and cell shape (for a review see [20]). Little is known about homologous signal transduction pathways in fission yeasts. One of the few examples is the recently identified Spm1/Pmh1-Mkh1 MAP-kinase cascade, whose defects enhance stress sensitivity, cause occasional cytogenesis defects and confer reduced fertility, but do not elicit mycelial growth associated with complete sterility $[21,22]$. The products of the sep $6^{+}$to sep $16^{+}$ genes might be involved in this or other signal transduction pathways, which have not been identified yet. In the eukaryotic cells the mitogen-activated protein kinases and their upstream regulators couple upstream signals to the modulation of co-ordination of various cellular processes and to the activation of the genes involved in these processes. Consistent with this view, two of the five cloned members or the $\operatorname{sep} 6^{+}$to $\operatorname{sep} 16^{+}$group show high degree of sequence homology with transcription regulators. $\operatorname{sep} 9^{+}$is a homologue of the Saccharomyces cerevisiae SPT8 [unpublished result], a protein that encodes a putative TATA-binding protein [23]. Inactivation of SPT8, also causes defects in growth, mating and sporulation. Cloning and sequencing of the rest of the sep genes is in progress.

Acknowledgements. We thank Lakatos Zoltánné, Pásztor Lajosné and Kerekes Ágnes for excellent technical assistance. The projects summarized in this paper were supported by numerous grants from the Hungarian Academy of Sciences, the Hungarian Scientific Research Fund and the National Committee for Technological Development.

\section{REFERENCES}

1. Sipiczki,M.: Phylogenesis of fission yeasts. Contradictions surrounding the origin of a century old genus. Antonie van Leeuwenhoek 68, 119-149 (1995).

2. Sipiczki,M.: Phylogenesis and evolution of fission yeasts. 14th International Specialised Symposium on Yeasts. Yeast Taxonomy: Theoretical and Practical Aspects. Smolenice, Czechoslovakia, Program and Abstracts, p. 35, 1990.

3. Sipiczki,M., Takeo,K., Yamaguchi,M., Yoshida,S., Miklos,I.: Environmentally controlled dimorphic cycle in a fission yeast. Microbiology 144, 1319-1330 (1998).

4. Sipiczki,M., Grallert,B., Miklós,I.: Mycelial and syncytial growth in Schizosaccharomyces pombe by novel septation mutations. J Cell Sci 104, 485-493 (1993). 
5. Grallert,A., Miklós,I., Sipiczki,M.: Division-site selection, cell separation, and formation of anucleate minicells in Schizosaccharomyces pombe mutants resistant to cell-wall lytic enzymes. Protoplasma 198, 218-229 (1997).

6. Grallert,A., Grallert,B., Zilahi,E., Szilágyi,Z., Sipiczki,M.: Eleven novel sep genes of Sch. pombe required for efficient cell separation and sexual differentiation. Yeast (in press)

7. Johnson,B.F., Calleja,G.B., Yoo,B.Y., Zuker,M., McDonald,I.J.: Cell division: key to cellular morphogenesis in the fission yeast, Schizosaccharomyces. Int Rev Cytol 75, 167-208 (1982).

8. Johnson,B.F., Yoo,B.Y., Calleja,G.B.: Cell division in yeasts: movement of organelles associated with cell plate growth of Schizosaccharomyces pombe. J Bacteriol 115, 358-366 (1973).

9. Sipiczki,M., Takeo,K., Grallert,A.: Growth polarity transition in a dimorphic fission yeast. Microbiology 144 (in press).

10. Mitchison,J.M., Nurse,P.: Growth in cell length in the fission yeast Schizosaccharomyces pombe. J Cell Sci 75, 357-376 (1985).

11. Miyata,H., Miyata,M., Johnson,B.F.: Patterns of extension growth of the fission yeast, Schizosaccharomyces pombe. Can J Microbiol 32, 528-530 (1986).

12. Miyata,H., Miyata,M., Johnson,B.F.: Patterns of end growth of the fission yeast Schizosaccharomyces pombe. Can J Microbiol 36, 390-394 (1990).

13. Sipiczki,M., Takeo,K.: The effect of caffeine on cell cycle progression and polar growth in Schizosaccharomyces pombe. Biologia, Bratislava 53, 291-296 (1998).

14. Ribar,B., Banrevi,A., Sipiczki,M.: sep $1^{+}$encodes a transcription-factor homologue of the HNF-3/forkhead DNA-binding-domain family in Schizosaccharomyces pombe. Gene 202, 1-5 (1997).

15. Grallert,A., Sipiczki,M.: Polarity, spatial organisation of cytoskeleton, and nuclear division in morphologically altered cells of Schizosaccharomyces pombe. Can J Microbiol 43, 991-998 (1997).

16. Grallert,A., Grallert,B., Ribar,B., Sipiczki,M.: Coordination of initiation of nuclear division and initiation of cell division in Schizosaccharomyces pombe: Genetic interactions of mutations. J Bacteriol 180, 892-900 (1998).

17. Chang,F., Nurse,P.: How fission yeast fission in the middle. Cell 84, 191-194 (1996).

18. Satterwhite,L.L., Pollard,T.D.: Cytokinesis. Curr Opin Cell Biol 4, 43-52 (1992).

19. Iino,Y., Yamamoto,M.: Mutants of Schizosaccharomyces pombe which sporulate in the haploid state. Mol Gen Genet 198, 416-421 (1985).

20. Madhani,H.D., Fink,G.R.: The riddle of MAP kinase signalling specificity. Trends Genet 14, 151-155 (1998).

21. Zaitsevskaya-Carter,T., Cooper,J.A.: Spm1, a stress-activated MAP kinase that regulates morphogenesis in S. pombe. EMBO J 16, 1318-1331 (1997).

22. Sengar,A.S., Markley,N.A., Marini,N.J., Young,D.: Mkh1, a MEK kinase required for cell wall integrity and proper response to osmotic and temperature stress in Schizosaccharomyces pombe. Mol Cell Biol 17, 35083519 (1997).

23. Eisenmann,D.M., Chapon,C., Roberts,S.M., Dollard,C., Winston,F.: The Saccharomyces SPT8 gene encodes a very acidic protein that is functionally related to SPT3 and TATA-binding protein. Genetics 137, 647-657 (1994). 